\title{
Update on the treatment of phenylketonuria: long-term safety and efficacy of sapropterin dihydrochloride
}

This article was published in the following Dove Press journal:

Research and Reports in Endocrine Disorders

26 May 2012

Number of times this article has been viewed

\section{Hilary Vernon ${ }^{1,2}$}

'McKusick-Nathans Institute of Genetic Medicine, Johns Hopkins University, ${ }^{2}$ Kennedy Krieger Institute, Baltimore, MD, USA
Correspondence: Hilary Vernon Blalock 1008, 600 North Wolfe Street, Baltimore, MD 2/287, USA

Tel + I $410955307 \mid$

Fax + 4106149246

Email hvernon I@jhmi.edu

\begin{abstract}
Phenylketonuria (PKU) is an inborn error of metabolism caused by a defect in the enzyme phenylalanine hydroxylase, which is responsible for converting phenylalanine to tyrosine. Untreated, this disorder will result in severe intellectual disability. However, with proper management, outcome is excellent. For many years, this disorder was managed exclusively with dietary measures which consisted of a phenylalanine-restricted diet. However, with the recent introduction of a stable, orally bioavailable form of tetrahydrobiopterin (BH4), the cofactor for phenylalanine hydroxylase, treatment in this disorder has been drastically altered. This stable form of BH4, sapropterin dihydrochloride, has a very good safety profile and is very effective in many patients with PKU in lowering plasma phenylalanine levels and allowing for liberalization of the phenylalanine-restricted diet. The introduction of BH4 has posed many new challenges in the treatment of PKU, including developing the best protocol to determine whether or not a patient will respond to $\mathrm{BH} 4$, and how to treat atypical populations including young children, fully affected, untreated adults, and pregnant patients. In this review, we will examine the history of treatment in PKU, the history of treatment with $\mathrm{BH} 4$, protocol options for determining if a patient is a drug responder, and considerations for treatment in special populations.
\end{abstract}

Keywords: sapropterin dihydrochloride, phenylketonuria, phenylalanine

\section{Introduction}

The identification and treatment of phenylketonuria (PKU) in many ways represents the "gold standard" for success in rational, biochemically based treatment for an inborn error of metabolism. Through proper treatment, the severe and devastating clinical effects of this disease can be ameliorated, allowing affected individuals to have normal cognition and long-term functioning. Until relatively recently, dietary management with restricted phenylalanine intake was the mainstay of treatment, however with introduction of tetrahydrobiopterin (BH4) supplementation, treatment strategies for many affected individuals have been drastically changed.

\section{Historical perspective}

Asbjorn Folling first described the association between elevated plasma phenylalanine and mental retardation in 1934 when he identified the presence of phenylacetic acid in the urine of two affected siblings. In 1953, George Jervis determined that the elevated phenylalanine levels were due to a deficiency of the enzyme phenylalanine hydroxylase. ${ }^{1}$ In the 1950 s Horst Bickel $^{2}$ developed the first phenylalanine-limited formula, and soon after it was noted that the best outcomes were in the earliest treated patients. In 1963, Robert Guthrie developed a blood test to screen for PKU in newborns, 
and a few years later it was introduced as a newborn screening test in Massachusetts. ${ }^{3}$ Several states followed quickly afterwards, and now every state in the United States of America has a newborn screening program which includes screening for PKU.

For two decades after treatment in PKU was introduced, it was thought that a low phenylalanine diet had to be maintained in infants and young children to avoid negative neurologic consequences. However, it became clear as early as the late 1970 s that elevated blood phenylalanine led to negative cognitive outcomes later in life, including loss of executive functioning skills, behavioral problems, and emotional difficulties including depression. ${ }^{4-7}$ Therefore, lifelong dietary management is recommended for optimal neurocognitive functioning.

In addition to the negative neurocognitive outcome on affected individuals, elevated maternal phenylalanine has long been recognized to be a fetal teratogen. Fetal consequences include microcephaly, intellectual disability, and congenital heart defects. These effects are related to the level of phenylalanine maintained during pregnancy, with higher levels causing the most severe consequences. ${ }^{8}$ Strict dietary control before and during pregnancy is crucial for optimal outcome.

Unfortunately, the phenylalanine-restricted diet, as with many specialized diets for inborn errors of metabolism, is less palatable than a regular diet and cumbersome to manage. This difficulty in maintaining the diet has led to a pervasive lack of compliance as patients enter adolescence. ${ }^{6}$

\section{Tetrahydrobiopterin therapy}

The cofactor for phenylalanine hydroxylase is BH4, which has its own multistep synthesis and recycling pathways (Figure 1). BH4 also serves as a cofactor for tyrosine hydroxylase, tryptophan hydroxylase, and nitric oxide synthase. Defects in the synthesis and recycling of BH4 can lead to hyperphenylalaninemia, as well as other substrate defects referable to central nervous system mediators. BH4, along with other neurotransmitter precursors, has been used to treat this family of disorders with success. ${ }^{9}$

Initially it was thought that $\mathrm{BH} 4$ therapy would only be effective in BH4 synthesis defects, however in 1999, Kure et al demonstrated that some patients with phenylalanine hydroxylase deficiency showed reduction in their plasma phenylalanine in response to BH4 therapy. ${ }^{10}$ Soon after, a stable, synthetic form of BH4, sapropterin dihydrochloride, was produced by BioMarin (Novato, CA), and clinical trials were undertaken.

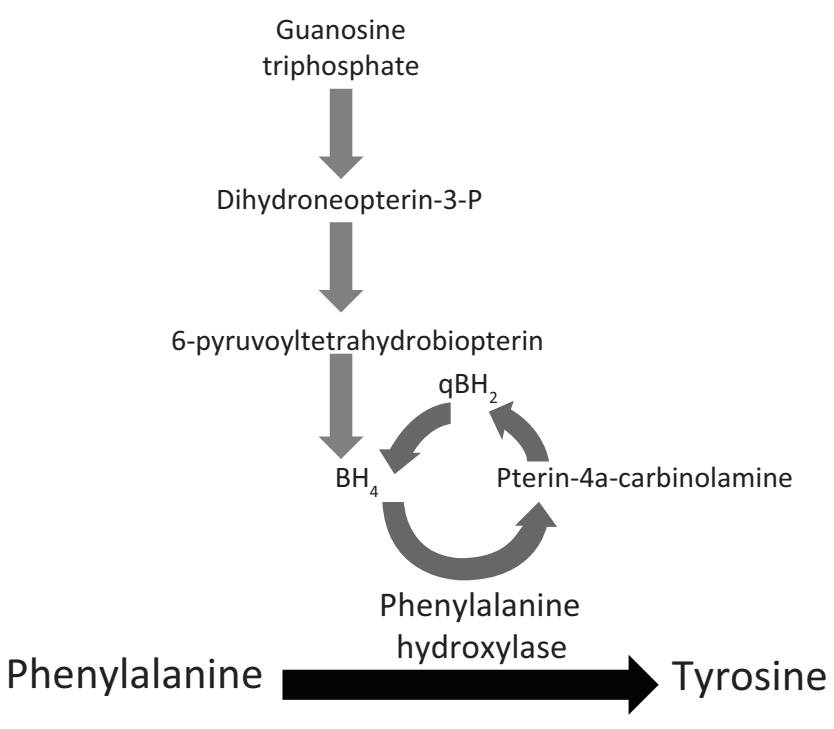

Figure I Phenylalanine is converted to tyrosine by the enzyme phenylalanine hydroxylase.

Note: Tetrahydrobiopterin (BH4) is the cofactor for this reaction, and has its own synthesis and regeneration pathways.

\section{Hyperphenylalaninemia, tetrahydrobiopterin, and neurotransmitter levels}

High phenylalanine levels are thought to interfere with the production of dopamine and catecholamines, a finding that has been observed in brain tissue of a mouse model for PKU. ${ }^{11}$ Reduced serotonin and catecholamine levels have also been observed in the erythrocyte membranes of poorly controlled PKU patients compared to well controlled PKU patients. ${ }^{12}$ These neurotransmitter abnormalities likely play a large role in the spectrum of pathology in untreated PKU seen throughout life. With introduction of BH4 to a phenylalaninerestricted diet, improvement in platelet serotonin production can be observed, indicating a role for BH4 in improving neurotransmitter abnormalities. ${ }^{13}$

\section{Tetrahydrobiopterin therapy: mechanism of action}

The responsiveness to $\mathrm{BH} 4$ in phenylalanine hydrolase (PAH) deficiency is due to the stabilization of certain PAH mutants by BH4. ${ }^{14} \mathrm{BH} 4$ appears to act as a chaperone by facilitating PAH folding into its active tetramer form, as opposed to the less active dimer form. ${ }^{15}$ Despite this relationship, some studies have demonstrated difficulty in assigning a genotypephenotype relationship for $\mathrm{BH} 4$ responsiveness in PKU. ${ }^{16}$ Some of this difficulty may be related to the fact that there are over 530 mutants described, and they are often found in a compound heterozygous state. ${ }^{17}$ Other confounding 
factors include the differences in sapropterin dihydrochloride doses and preparations used in various studies, different cutoff points for responsiveness, and lack of strict control over dietary manipulation or assessment.

However, with careful, controlled studies a genotypephenotype relationship has been demonstrated for several common genotypes. ${ }^{18}$ Utz et al described several genotypes associated with responsiveness in two or more patients including p.Y414C and p.I65T, and several genotypes associated with non-responsiveness in two or more patients including p.R408W/IVS12nt1 G > A and p.R408W/p.A104D. ${ }^{17}$ Generally, patients with alleles with higher residual activity were more responsive, however, this was not an absolute rule and there were notable exceptions. This relationship to residual enzyme activity was also observed by Dubrowski et al in the Turkish PKU population. ${ }^{19}$

\section{Clinical trials}

The first three clinical trials on sapropterin dihydrochloride were run sequentially, with subsequent study populations being selected from previous study populations. The study participants were aged over 8 years old, and had uncontrolled or poorly controlled plasma phenylalanine levels. The first clinical trial (PKU-001) was an open-label Phase II study that tested patients for responsiveness to sapropterin dihydrochloride defined as a greater than $30 \%$ decrease in plasma Phe levels after taking sapropterin dihydrochloride for 8 days at a dose of $10 \mathrm{mg} / \mathrm{kg} /$ day. In this study 96 of 485 patients were classified as "responders". ${ }^{20}$ The next trial (PKU-003) was an open-label extension study that enrolled 89 of the 96 responders from the first study, which showed a consistent lowering of plasma phenylalanine levels over a 6-week period of administration of sapropterin dihydrochloride at $10 \mathrm{mg} /$ $\mathrm{kg} /$ day. $^{21}$

The subsequent study, PKU-004, was an extension of PKU-003, and looked at efficacy over a 22-week period at a range of effective sapropterin dihydrochloride doses ranging from $5 \mathrm{mg} / \mathrm{kg} /$ day to $20 \mathrm{mg} / \mathrm{kg}$ /day in previously determined responders. This study observed dose-dependent reductions in plasma phenylalanine concentrations. During this study, no severe or serious adverse events were considered to be related to sapropterin dihydrochloride, and no adverse event led to discontinuation of the medication. ${ }^{22}$

The next clinical trial, PKU-006, was a Phase III study that looked at the safety and efficacy of sapropterin dihydrochloride at $20 \mathrm{mg} / \mathrm{kg} / \mathrm{day}$. In the first part of the study, 90 children with PKU aged 4-12 years who were well controlled on Phe-restricted diets were given an 8-day trial of sapropterin dihydrochloride to determine responsiveness. Responsiveness in this study was defined as a greater than $30 \%$ decrease in plasma phenylalanine levels and a plasma phenylalanine level less than $300 \mu \mathrm{mol} / \mathrm{L}$. Fifty of the 90 children were determined to be responders. Most of the responders were enrolled in the second part of the study that assessed dietary tolerance to increased dietary phenylalanine intake. A significant increase in tolerance to dietary phenylalanine intake was observed. ${ }^{23}$ No serious or severe adverse events related to the medication were observed.

The most recent clinical trial, PKU-008, was a Phase IIIb trial that evaluated the long-term safety and efficacy of sapropterin dihydrochloride use in previously determined sapropterin dihydrochloride responders enrolled in prior trials. These patients had an average sapropterin dihydrochloride exposure of 659 days, and an average daily dose of $16 \mathrm{mg} / \mathrm{kg} .{ }^{24}$ Patients were monitored every 3 months for adverse event occurrences, and with laboratory and clinical evaluations. Twenty-five percent of patients had a temporary, but self-limited, drop in plasma phenylalanine below the lower recommended limit for a PKU patient $(120 \mu \mathrm{mol} / \mathrm{L})$. Temporary decreases in neutrophil count were also reported. Low platelet count was also reported in several patients, but the relationship to sapropterin dihydrochloride is unclear. ${ }^{24}$

One severe event occurred and was possibly related to sapropterin dihydrochloride exposure, which was difficulty concentrating and mood swings. One serious event occurred that was considered to be probably related to sapropterin dihydrochloride exposure, and resulted in hospitalization for gastroesophageal reflux disease. Three participants discontinued sapropterin dihydrochloride due to adverse events thought to be possibly or probably related to the drug, including difficulty concentrating, decreased platelet count, or diarrhea. The most frequent adverse events thought to be related to sapropterin dihydrochloride exposure were vial gastroenteritis, vomiting, and headache, each occurring in $4.5 \%$ of patients. No deaths were reported. ${ }^{24}$

Overall, these studies indicate a good safety profile for sapropterin dihydrochloride, and significant clinical effect with a statistically significant decrease in plasma phenylalanine levels and increase in dietary phenylalanine intake in sapropterin dihydrochloride responders.

\section{Determining responsiveness in a clinical setting}

The determination of whether or not a patient will be a sapropterin dihydrochloride responder is not entirely straightforward. Several strategies have been suggested 
including sapropterin dihydrochloride loading trials or more extended testing for a $20 \%-30 \%$ decrease in plasma phenylalanine from pretreatment levels. Other groups also consider increased tolerance to dietary phenylalanine as responder criteria. Some groups propose to use these strategies and to include knowledge of genotype/phenotype correlations. It is also generally accepted that patients with higher baseline levels of phenylalanine (ie, those with "classical" PKU with a baseline phenylalanine of $>1200 \mu \mathrm{Mmol}$ ) are less likely to respond to sapropterin dihydrochloride than those with variant or benign forms of PKU..$^{20,25}$

The BH4-loading test has been used in Europe for more than 30 years and was initially used to discriminate patients with PKU from those with defects in $\mathrm{BH} 4$ synthesis or regeneration. ${ }^{26}$ Different strategies have been proposed for the BH4-loading test, but generally involve a 48-72-hour protocol wherein the patient's baseline phenylalanine level is obtained on a phenylalanine-unrestricted diet, and the patient is usually loaded with $20 \mathrm{mg} / \mathrm{kg}$ of sapropterin dihydrochloride. The blood phenylalanine is monitored every 8 hours for the duration of the study, and responsiveness is determined by a set decrease in the plasma phenylalanine level, usually $>30 \%{ }^{26}$ The advantages of this test include limiting the variability of dietary influence on the results, and relatively short time to obtain results. Disadvantages include the cost of potentially having to hospitalize a patient to obtain all of the appropriate, timed measurements, and the potential of missing "slow sapropterin dihydrochloride responders" of which a few have been reported. ${ }^{27}$

Some groups advocate a longer trial to determine sapropterin dihydrochloride responsiveness, similar to those employed in the clinical trials described above (Figures 2 and 3). In these trials, a patient's baseline phenylalanine level is determined, and the patient is started on a $10 \mathrm{mg} / \mathrm{kg} /$ day or $20 \mathrm{mg} / \mathrm{kg} /$ day dose of sapropterin dihydrochloride. Blood phenylalanine levels are measured on a weekly basis, and responsiveness is determined usually by a decrease from baseline plasma phenylalanine by $>30 \% .{ }^{25}$ Advantages of this testing method include the ability to conduct the testing easily on an outpatient (as opposed to an inpatient basis) and the ability to pick up slow responders. Disadvantages include susceptibility to dietary manipulation, either intentional or unintentional, and susceptibility to other environmental factors including intercurrent illness, etc.

Utz et al have recently published a trial to determine sapropterin dihydrochloride responsiveness using a double-blind, placebo-controlled test in order to overcome some of the

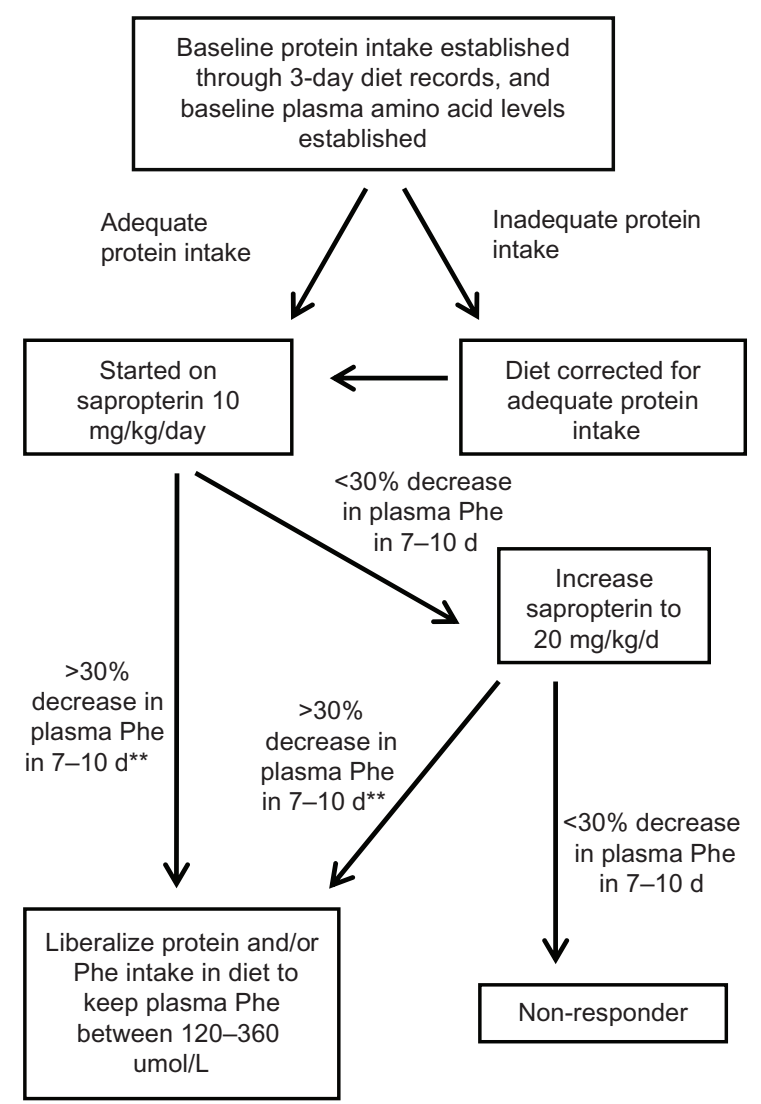

Figure 2 Outpatient clinical protocol for determining sapropterin dihydrochloride responsiveness used at the McKusick-Nathans Institute of Genetic Medicine, Johns Hopkins University.

Note: **Or decrease to treatment range.

susceptibility to dietary manipulation inherent in the longer trials. In this trial, patients were tested for 4 weeks for responsiveness to placebo or sapropterin dihydrochloride in a double-blinded fashion. ${ }^{18}$ The response to sapropterin dihydrochloride was defined as a $>20 \%$ decrease in the plasma phenylalanine during both weeks of administration of medication versus the prior week (control or baseline). This group also included PAH genotyping in their study in order to determine any relationship between genotype and responsiveness. Some general relationships were found (see discussion on genotype/phenotype relationship).

Overall each method has its advantages and disadvantages, and ultimately a combination of these methods best suited to meet the needs of the individual patient or institution may prove to be the most utilitarian.

\section{Direct measurements of clinical outcome}

While it is well accepted that lowering phenylalanine levels in patients with PKU results in improved neurocognitive 
Patient 1: response to sapropterin

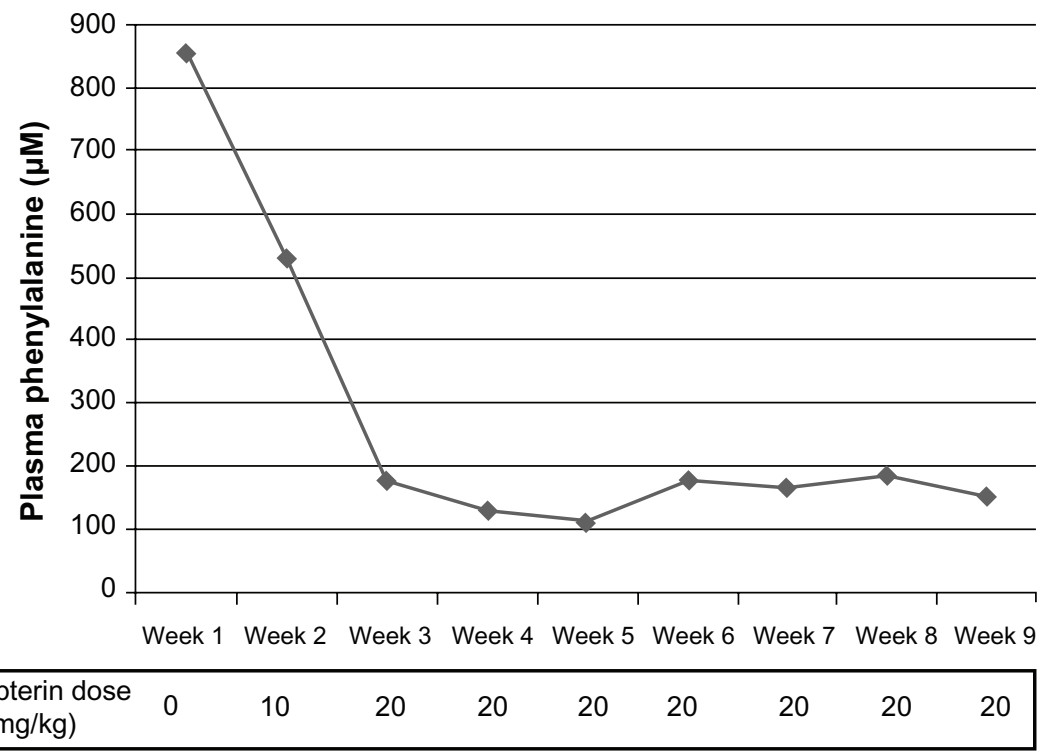

Figure 3 Data collected from a patient with phenylketonuria who was determined to be a sapropterin dihydrochloride responder.

Notes: This patient had a $38 \%$ reduction in her plasma phenylalanine from baseline on $10 \mathrm{mg} / \mathrm{kg} /$ day of sapropterin dihydrochloride, and a $79 \%$ reduction in her plasma phenylalanine from baseline on $20 \mathrm{mg} / \mathrm{kg} / \mathrm{day}$ of sapropterin dihydrochloride. This was a sustained response. Her diet was eventually liberalized (data not shown).

outcome, the direct relationship between sapropterin use and neurocognitive status has not been made (Sapropterin Dihydrochloride Package Insert; BioMarin). This seems like a logical correlation, however formal studies would be helpful in solidifying this relationship.

In many responder patients, administration of sapropterin dihydrochloride has resulted in the ability to have a more phenylalanine-liberal diet. ${ }^{25}$ While studies on the social and psychological implications of a less restrictive PKU diet have not been formally done, it seems that this would be a clear benefit to this drug.

\section{Young children}

The initial clinical trials of sapropterin dihydrochloride did not include participants younger than 4 years old. Therefore, safety and efficacy in young children has not been "officially" evaluated in a clinical trial, leading to some hesitancy in using sapropterin dihydrochloride in this population. Burton et al recently published six case reports treating young patients aged 7 months to 4 years. ${ }^{28}$ They report that sapropterin dihydrochloride was safe, generally well tolerated, and clinically effective in reducing blood phenylalanine levels or increasing dietary phenylalanine tolerance. Gastrointestinal side effects (diarrhea) were reported in one patient.

Our own clinic has had similar observations, and found this medication to be generally well tolerated and clinically effective in several young patients younger than 4 years old.

\section{Pregnant patients}

The US Food and Drug Administration approved the use of sapropterin dihydrochloride in pregnancy with the condition that a pregnancy registry for women with maternal PKU using sapropterin dihydrochloride alone be developed. ${ }^{29}$ Teratogenicity studies with sapropterin were conducted in rats and rabbits. No significant evidence of teratogenesis was found in either species, although in the rabbit teratogenicity study, there was a statistically insignificant increase in the incidence of holoprosencephaly (Sapropterin Dihydrochloride Package Insert; BioMarin).

Case reports of sapropterin dihydrochloride use in pregnancy are rare in the literature. However, Koch et al reported the use of this drug in a single pregnancy in a mother with classical PKU, and a good pregnancy outcome with a child with above average IQ at 4 years of age. ${ }^{28,89}$ Koch recommends an initial lower dose of 50 to $100 \mathrm{mg}$ /day of sapropterin dihydrochloride in the first trimester of pregnancy with incremental increase in later trimesters, however clinical trials of dosing in pregnancy have not been done. ${ }^{29}$ Our own clinic has had positive outcomes in treating several pregnancies affected by maternal PKU with sapropterin dihydrochloride.

\section{Older, classically affected patients}

Reports of treating older, classically affected, and untreated PKU patients with sapropterin dihydrochloride are sparse. 
However, taking into account the published percentages of at least $10 \%$ of classical PKU patients being sapropterin dihydrochloride responders, biochemical improvements would be expected in certain fully affected adults. ${ }^{20,25}$ In addition, positive neurocognitive effects have been seen in previously untreated adult PKU patients placed on a phenylalaninerestricted diet. ${ }^{31,32}$ Our own clinic has had experience in treating a fully affected, previously untreated adult with PKU who was found to be a sapropterin dihydrochloride responder. This adult had noticeable improvements in behavior, nutrition, and overall well-being. Therefore we believe that testing this population for sapropterin dihydrochloride responsiveness is worthwhile.

\section{Conclusion}

Introduction of sapropterin dihydrochloride has changed the standard of care in patients with PKU. Challenges in introduction of this medication include determining the best method for testing for sapropterin dihydrochloride responsiveness in affected individuals, as well as introduction into nonstandard populations including pregnant women, young children, and previously untreated adults. Future studies into long-term neurocognitive outcome will ultimately be the greatest marker for success of this medication, and are yet to be published; however positive effects include lowering of plasma phenylalanine levels and liberalization of diet in many sapropterin dihydrochloride-responsive individuals. Despite challenges, the evidence overwhelmingly suggests that this is a relatively safe, well tolerated medication that will lower plasma phenylanine levels in sapropterin dihydrochlorideresponsive PKU patients.

\section{Disclosure}

The author reports no conflicts of interest in this work.

\section{References}

1. Jervis GA. Phenylpyruvic oligophrenia deficiency of phenylalanineoxidizing system. Proc Soc Exp Biol Med. 1953;82(2):514-515.

2. Bickel $\mathrm{H}$. The effects of a phenylalanine-free and phenylalanine-poor diet in phenylpyruvic oligophrenis. Exp Med Surg. 1954;12(1):114-117.

3. Guthrie R, Susi A. A simple phenylalanine method for detecting phenylketonuria in large populations of newborn infants. Pediatrics. 1963;32(3):338-343.

4. Smith I, Lobascher ME, Stevenson JE, et al. Effect of stopping low-phenylalanine diet on intellectual progress of children with phenylketonuria. Br Med J. 1978;2(6139):723-726.

5. VanZutphen KH, Packman W, Sporri L, et al. Executive functioning in children and adolescents with phenylketonuria. Clin Genet. 2007;72(1):13-18.

6. Koch R, Burton B, Hoganson G, et al. Phenylketonuria in adulthood: a collaborative study. J Inherit Metab Dis. 2002;25(5):333-346.

7. Levy HL, Waisbren SE. PKU in adolescents: rationale and psychosocial factors in diet continuation. Acta Paediatr. 1994;407:92-97.
8. Koch R, Hanley W, Levy H, et al. The Maternal Phenylketonuria International Study 1984-2002. Pediatrics. 2003; 112(6 pt 2): 1523-1529.

9. Hyland K, Cotton RH, Thony B, Blau N. Disorders of tetrahydrobiopterin and related biogenic amines. In: Valle D, Beaudet AL, Vogelstein B, Kinzler KW, Antonarakis SE, Ballabio A, editors. The Online Metabolic and Molecular Bases of Inherited Disease. Available at http://www. ommbid.com/. Accessed Sep 2012.

10. Kure S, Hou DC, Ohura T, et al. Tetrahydrobiopterin-responsive phenylalanine hydroxylase deficiency. J Pediatr. 1999;135(3): 375-378.

11. Curtius H, Wiederwieser C, Viscontini G, Leimbacher N, Wegman H, Schmidt H. Serotonin and dopamine synthesis in phenylketonuria. $A d v$ Exp Med Biol. 1981;133:277-291.

12. Puglisi-Allegra S, Cabib S, Pascucci T, Ventura R, Cali F, Romano V. Dramatic brain aminergic deficit in a genetic mouse model of phenylketonuria. Neuroreport. 2000;11(6):1361-1364.

13. Ormazabal A, Vilaseca MA, Pérez-Dueñas B, et al. Platelet serotonin concentrations in PKU patients under dietary control and tetrahydrobiopterin treatment. J Inherit Metab Dis. 2005;28(6):863-870.

14. Erlandsen H, Pey AL, Gamez A, et al. Correction of kinetic and stability defects by tetrahydrobiopterin in phenylketonuria patients with certain phenylalanine hydroxylase mutations. Proc Natl Acad Sci U SA. 2004;101(48):16902-16908.

15. Bjorgo E, de Carvalho RM, Flatmark T. A comparison of kinetic and regulatory properties of the tetrameric and dimeric forms of wildtype and Thr427-->Pro mutant human phenylalanine hydroxylase: contribution of the flexible hinge region Asp425-Gln429 to the tetramerization and cooperative substrate binding. Eur J Biochem. 2001;268(4):997-1005.

16. Karacic I, Meili D, Sarnavka V, et al. Genotype predicted tetrahydrobiopterin (BH4)-responsiveness and molecular genetics in Croatian patients with phenylalanine hydroxylase (PAH) deficiency. Mol Genet Metab. 2009;97(3):165-171.

17. Sarafoglou K, Hoffman G, Roth K. Pediatric Endocrinology and Inborn Errors of Metabolism. New York, NY: McGraw-Hill; 2008.

18. Utz J, Lorentz C, Markowitz D, et al. START, a double blind placebocontrolled pharmacogenetic test of responsiveness to sapropterin dihydrochloride in phenylketonuria patients. Mol Genet Metab. 2012;105(2):193-197.

19. Dubrowski S, Heintz C, Miller T, et al. Molecular genetics and impact of residual in vitro phenylalanine hydroxylase activity on tetrahydrobiopterin responsiveness in Turkish PKU population. Mol Genet Metab. 2011;102(2):116-121.

20. Burton BK, Grange DK, Milanowski G, et al. The response of patients with phenylketonuria and elevated serum phenylalanine to treatment with oral sapropterin dihydrochloride (6R-tetrahydrobiopterin): a phase II, multicentre, open-label, screening study. J Inherit Metab Dis. 2007;30(5):700-777.

21. Levy H, Milanowski A, Chakrapani A, et al; Sapropterin Research Group. Efficacy of sapropterin dihydrochloride (tetrahydrobiopterin, 6R-BH4) for reduction of phenylalanine concentration in patients with phenylketonuria: a phase III randomized clinical placebo-controlled study. Lancet. 2007;370(9586):504-510.

22. Lee P, Treacy E, Crombex W, et al. Safety and efficacy of 22 weeks of treatment with sapropterin dihydrochloride in patients with phenylketonuria. Am J Med Genet. 2008;146A(22):2851-2859.

23. Tretz FK, Burtin BK, Longo N, et al. Efficacy of sapropterin dihydrochloride in increasing phenylalanine tolerance in children with phenylketonuria: a Phase III, randomized, double-blind, placebo controlled study. J Pediatr. 2009;154(5):700-707.

24. Burton B, Nowacka M, Hennermann JB, et al. Safety of extended treatment with sapropterin dihydrochloride in patients with phenylketonuria: Results of a phase 3b study. Mol Genet Metab. 2011; 103(4):315-322.

25. Vernon HJ, Koerner CB, Johnson MR, Bergner A, Hamosh A. Introduction of sapropterin dihydrochloride as standard of care in patients with phenylketonuria. Mol Genet Metab. 2010;100(3):229-233. 
26. Blau N, Hennermann J, Langenbeck U, Lickter-Konecki U. Diagnosis, classification and genetics of phenylketonuria and tetrahydrobiopterin (BH4) deficiencies. Mol Genet Metab. 2011;104 Suppl:S2-S9.

27. Fiege B, Bonafe L, Ballhausen D, et al. Extended tetrahydrobiopterin loading test in the diagnosis of cofactor-responsive phenylketonuria: a pilot study. Mol Genet Metab. 2005;86(S1):91-95.

28. Burton BK, Adams DJ, Grange DK, et al. Tetrahydrobiopterin therapy for phenylketonuria in infants and young children. J Pediatr. 2011 158(3):410-415.

29. Koch R. Maternal phenylketonuria and tetrahydrobiopterin. Pediatrics. 2008;122(6):1367-1368.
30. Koch R, Moseley K, Guttler F. Tetrahydrobiopterin and maternal PKU. Mol Genet Metab. 2005;86(Suppl 1):S139-S141.

31. Lee PJ, Amos A, Robertson L, et al. Adults with late diagnosed PKU and severe challenging behaviour: a randomised placebo-controlled trial of a phenylalanine-restricted diet. J Neurol Neurosurg Psychiatry. 2009;80(6):631-635.

32. Grosse SD. Late-treated phenylketonuria and partial reversibility of intellectual impairment. Child Dev. 2010;81(1):200-211.

Research and Reports in Endocrine Disorders

\section{Publish your work in this journal}

Research and Reports in Endocrine Disorders is an international, peerreviewed, open access journal publishing original research, reports, reviews and commentaries on all areas of endocrinology, endocrine disorders and therapeutic interventions. The manuscript management system is completely online and includes a very quick and fair peer-review system. Visit http://www.dovepress.com/testimonials.php to read real quotes from published authors.

Submit your manuscript here: http://www.dovepress.com/research-and-reports-in-endocrine-disorders-journal 